\title{
Research Article \\ Remarks on Generalized Derivations in Prime and Semiprime Rings
}

\author{
Basudeb Dhara \\ Department of Mathematics, Belda College, Belda, Paschim Medinipur 721424, India \\ Correspondence should be addressed to Basudeb Dhara, basu_dhara@yahoo.com
}

Received 15 August 2010; Accepted 28 November 2010

Academic Editor: Hans Keiding

Copyright (C 2010 Basudeb Dhara. This is an open access article distributed under the Creative Commons Attribution License, which permits unrestricted use, distribution, and reproduction in any medium, provided the original work is properly cited.

Let $R$ be a ring with center $Z$ and $I$ a nonzero ideal of $R$. An additive mapping $F: R \rightarrow R$ is called a generalized derivation of $R$ if there exists a derivation $d: R \rightarrow R$ such that $F(x y)=$ $F(x) y+x d(y)$ for all $x, y \in R$. In the present paper, we prove that if $F([x, y])= \pm[x, y]$ for all $x, y \in I$ or $F(x \circ y)= \pm(x \circ y)$ for all $x, y \in I$, then the semiprime ring $R$ must contains a nonzero central ideal, provided $d(I) \neq 0$. In case $R$ is prime ring, $R$ must be commutative, provided $d \neq 0$. The cases (i) $F([x, y]) \pm[x, y] \in Z$ and (ii) $F(x \circ y) \pm(x \circ y) \in Z$ for all $x, y \in I$ are also studied.

\section{Introduction}

Let $R$ be an associative ring. The center of $R$ is denoted by $Z$. For $x, y \in R$, the symbol $[x, y]$ will denote the commutator $x y-y x$ and the symbol $x \circ y$ will denote the anticommutator $x y+y x$. We will make extensive use of basic commutator identities $[x y, z]=[x, z] y+x[y, z]$, $[x, y z]=[x, y] z+y[x, z]$. An additive mapping $d$ from $R$ to $R$ is called a derivation of $R$ if $d(x y)=d(x) y+x d(y)$ holds for all $x, y \in R$. An additive mapping $g$ from $R$ to $R$ is called a generalized derivation of $R$ if there exists a derivation $d$ from $R$ to $R$ such that $g(x y)=$ $g(x) y+x d(y)$ holds for all $x, y \in R$. Obviously, every derivation is a generalized derivation of $R$. Thus, generalized derivation covers both the concept of derivation and left multiplier mapping. A mapping $F$ from $R$ to $R$ is called centralizing on $S$ where $S \subseteq R$, if $[F(x), x] \in Z$ for all $x \in S$.

Over the last several years, a number of authors studied the commutativity in prime and semiprime rings admitting derivations and generalized derivations. In [1], Daif and Bell proved that if $R$ is a semiprime ring with a nonzero ideal $K$ and $d$ is a derivation of $R$ such that $d([x, y])= \pm[x, y]$ for all $x, y \in K$, then $K$ is central ideal. In particular, if $K=R$, then $R$ 
is commutative. Recently, Quadri et al. [2] generalized this result replacing derivation $d$ with a generalized derivation in a prime ring $R$. More precisely, they proved the following.

Let $R$ be a prime ring and $I$ a nonzero ideal of $R$. If $R$ admits a generalized derivation $F$ associated with a nonzero derivation $d$ such that any one of the following holds: (i) $F([x, y])=[x, y]$ for all $x, y \in I$, (ii) $F([x, y])=-[x, y]$ for all $x, y \in I$, (iii) $F(x \circ y)=(x \circ y)$ for all $x, y \in I$; (iv) $F(x \circ y)=-(x \circ y)$ for all $x, y \in I$, then $R$ is commutative.

In the present paper, we study all these cases in semiprime ring.

\section{Main Results}

We recall some known results on prime and semiprime rings.

Lemma 2.1 (see [3, Lemma 1.1.5] or [1, Lemma 2]). (a) If $R$ is a semiprime ring, the center of a nonzero one-sided ideal is contained in the center of $R$, in particular, any commutative one-sided ideal is contained in the center of $R$.

(b) If $R$ is a prime ring with a nonzero central ideal, then $R$ must be commutative.

Lemma 2.2 (see [1, Lemma 1]). Let $R$ be a semiprime ring and I a nonzero ideal of $R$. If $z \in R$ and $z$ centralizes $[I, I]$, then $z$ centralizes $I$.

Lemma 2.3 (see [4, Theorem 3]). Let $R$ be a semiprime ring and $U$ a nonzero left ideal of $R$. If $R$ admits a derivation $d$ which is nonzero on $U$ and centralizing on $U$, then $R$ contains a nonzero central ideal.

Now we begin with the theorem.

Theorem 2.4. Let $R$ be a semiprime ring, I a nonzero ideal of $R$ and $F$ a generalized derivation of $R$ associated with a derivation $d$ of $R$ such that $d(I) \neq 0$. If $F([x, y])= \pm[x, y]$ for all $x, y \in I$, then $R$ contains a nonzero central ideal.

Proof. By our assumption, we have that

$$
F([x, y])= \pm[x, y]
$$

for all $x, y \in I$. If $F(I)=0$, then we find that $[x, y]=0$ for all $x, y \in I$, that is, $I$ is commutative. Then, by Lemma 2.1, $I \subseteq Z$ and thus we obtain our conclusion.

Next assume that $F(I) \neq 0$. Putting $y=y x$ in (2.1), we get that

$$
F([x, y] x)= \pm[x, y] x
$$

Since $F$ is a generalized derivation of $R$ associated with a derivation $d$ of $R,(2.2)$ gives

$$
F([x, y]) x+[x, y] d(x)= \pm[x, y] x
$$

Using (2.1), it reduces to

$$
[x, y] d(x)=0
$$


for all $x, y \in I$. Now putting $y=d(x) y$ in (2.4), we get

$$
0=[x, d(x) y] d(x)=d(x)[x, y] d(x)+[x, d(x)] y d(x)
$$

Using (2.4), it gives

$$
0=[x, d(x)] y d(x)
$$

for all $x, y \in I$. Now we put $y=y x$ in (2.6) and obtain that

$$
0=[x, d(x)] y x d(x)
$$

for all $x, y \in I$. Right multiplying (2.6) by $x$ and then subtracting from (2.7), we get

$$
0=[x, d(x)] y[x, d(x)]
$$

for all $x, y \in I$. This implies for all $x \in I$ that $([x, d(x)] I)^{2}=0$ and so $[x, d(x)] I=0$, forcing $[x, d(x)] \in I \cap \operatorname{Ann}(I)=0$. Then by Lemma 2.3, $R$ contains a nonzero central ideal.

Corollary 2.5. Let $R$ be a prime ring, I a nonzero ideal of $R$ and $F$ a generalized derivation of $R$. If $F([x, y])= \pm[x, y]$ for all $x, y \in I$, then $R$ is commutative or $F(x)= \pm x$ for all $x \in I$.

Proof. Let $d$ be the associated derivation of $F$. By Theorem 2.4, we conclude that either $d(I)=$ 0 or $R$ is commutative. Assume that $R$ is not commutative. Then $d(I)=0$. Since $R$ is a prime ring, $d(I)=0$ implies $d(R)=0$ and hence $F(x y)=F(x) y$ for all $x, y \in R$. Set $G(x)=F(x) \mp x$ for all $x \in R$. Then $G(x y)=G(x) y$ for all $x \in R$. Now, our assumption $F([x, y])= \pm[x, y]$ gives $F(x) y-F(y) x= \pm(x y-y x)$, that is, $G(x) y-G(y) x=0$ for all $x, y \in I$. Thus using $G(x) y=G(y) x$, we have $G(x) y z=G(y) x z=G(x z) y=G(x) z y$, that is, $G(x)[y, z]=0$ for all $x, y, z \in I$. Thus $0=G(I)[I, I]=G(I R)[I, I]=G(I) R[I, I]$. Since $R$ is prime, this implies $G(I)=0$ or $I$ is commutative. By Lemma 2.1, $I$ commutative implies that $R$ is commutative, a contradiction. Thus $G(I)=0$ which gives $G(x)=F(x) \mp x=0$ for all $x \in I$.

Theorem 2.6. Let $R$ be a semiprime ring, $I$ a nonzero ideal of $R$ and $F$ a generalized derivation of $R$ associated with a derivation $d$ of $R$ such that $d(I) \neq 0$. If $F(x \circ y)= \pm(x \circ y)$ for all $x, y \in I$, then $R$ contains a nonzero central ideal.

Proof. If $F(I)=0$, then by our assumption we have that $x \circ y=0$, that is, $x y+y x=0$ for all $x, y \in I$. This implies that $x(y z)=-(y z) x=-y(z x)=y(x z)=(y x) z=-(x y) z$ for all $x, y, z \in I$ and so $2 I^{3}=0$, forcing $2 I=0$. Therefore, for all $x, y \in I, x y+y x=0$ gives $x y=y x$, that is, $I$ is commutative. Then by Lemma $2.1, I \subseteq Z$ and thus we obtain our conclusion.

Next assume that $F(I) \neq 0$. Then for any $x, y \in I$, we have

$$
F(x y+y x)= \pm(x y+y x) .
$$


Since $F$ is a generalized derivation associated with a derivation $d$, above expression yields

$$
F(x) y+x d(y)+F(y) x+y d(x)= \pm(x y+y x) .
$$

Putting $y=y x$ in (2.10), we have

$$
F(x) y x+x(d(y) x+y d(x))+(F(y) x+y d(x)) x+y x d(x)= \pm\left(x y x+y x^{2}\right)
$$

Right multiplying (2.10) by $x$ and then subtracting from (2.11), we get

$$
x y d(x)+y x d(x)=0
$$

for all $x, y \in I$. Replacing $y$ with $d(x) y$ in (2.12) and then again using (2.12) we find that

$$
[x, d(x)] y d(x)=0
$$

Again replacing $y$ with $y x$ in (2.13) and then using (2.13) we obtain

$$
[x, d(x)] y[x, d(x)]=0
$$

for all $x, y \in I$, which is the same identity as (2.8) in the proof of Theorem 2.4. Thus by the same argument as in the proof of Theorem 2.4 , we conclude that $R$ contains a nonzero central ideal.

Corollary 2.7. Let $R$ be a prime ring, I a nonzero ideal of $R$ and $F$ a generalized derivation of $R$. If $F(x \circ y)= \pm(x \circ y)$ for all $x, y \in I$, then $R$ is commutative or $F(x)= \pm x$ for all $x \in I$.

Proof. Let $d$ be the associated derivation of $F$. By Theorem 2.6, we conclude that either $d(I)=$ 0 or $R$ is commutative. If $R$ is not commutative, then $d(I)=0$. Since $R$ is a prime ring, $d(I)=$ 0 implies $d(R)=0$ and hence $F(x y)=F(x) y$ for all $x, y \in R$. Set $G(x)=F(x) \mp x$ for all $x \in R$. Then $G(x y)=G(x) y$ for all $x \in R$. Now, our assumption $F(x \circ y)= \pm(x \circ y)$ gives $F(x) y+F(y) x= \pm(x y+y x)$, that is, $G(x) y+G(y) x=0$ for all $x, y \in I$. Thus using $G(x) y=-G(y) x$, we have $G(x) y z=-G(y) x z=G(x z) y=G(x) z y$, that is, $G(x)[y, z]=0$ for all $x, y, z \in I$. Thus $0=G(I)[I, I]=G(I R)[I, I]=G(I) R[I, I]$. Since $R$ is prime, this implies $G(I)=0$ or $I$ is commutative. By Lemma 2.1, $I$ commutative implies that $R$ is commutative, a contradiction. Therefore, $G(I)=0$ and hence $G(x)=F(x) \mp x=0$ for all $x \in I$.

Theorem 2.8. Let $R$ be a semiprime ring with center $Z \neq\{0\}, I$ a nonzero ideal of $R$ and $F$ a generalized derivation of $R$ associated with a derivation d of $R$. If $F([x, y]) \pm[x, y] \in Z$ for all $x, y \in I$, then $\operatorname{Id}(Z) \subseteq Z$.

Proof. We have

$$
F([x, y]) \pm[x, y] \in Z
$$


for all $x, y \in I$. Since $Z \neq\{0\}$, we may choose $0 \neq z \in Z$. Then $y z \in I$ for any $y \in I$. Now we replace $y$ with $y z$ in (2.15) and then we get

$$
\begin{aligned}
F([x, y] z) \pm[x, y] z & =F([x, y]) z+[x, y] d(z) \pm[x, y] z \\
& =\{F([x, y]) \pm[x, y]\} z+[x, y] d(z) \in Z .
\end{aligned}
$$

By (2.15), we have $[x, y] d(z) \in Z$ for all $x, y \in I$. Since $d(z) \in Z$, this gives that for any $r \in R,[r,[x, y] d(z)]=0$ which implies $[r d(z),[x, y]]=0$ for all $x, y \in I$. By Lemma 2.2, $[r d(z), x]=0$ for all $x \in I$. Since $d(z) \in Z$, this gives $[r, x d(z)]=0$ for all $r \in R$ and for all $x \in I$. Thus, $x d(z) \in Z$, that is, $\operatorname{Id}(Z) \subseteq Z$.

Corollary 2.9. Let $R$ be a prime ring with center $Z \neq\{0\}, I$ a nonzero ideal of $R$ and $F$ a generalized derivation of $R$ associated with a derivation $d$. If $d(Z) \neq\{0\}$ and $F([x, y]) \pm[x, y] \in Z$ for all $x, y \in I$, then $R$ is commutative.

Proof. Since $d(Z) \subseteq Z$ and $Z$ contains no nonzero elements which are zero divisors, we have from Theorem 2.8 that $I \subseteq Z$. Then by Lemma 2.1(b), we obtain our conclusion.

Theorem 2.10. Let $R$ be a semiprime ring with center $Z \neq\{0\}, I$ a nonzero ideal of $R$ and $F a$ generalized derivation of $R$ associated with a derivation d of $R$. If $F(x \circ y) \pm(x \circ y) \in Z$ for all $x, y \in I$, then $\operatorname{Id}(Z) \subseteq Z$.

Proof. We have

$$
F(x \circ y) \pm(x \circ y) \in Z
$$

for all $x, y \in I$. Since $Z \neq\{0\}$, we choose $0 \neq z \in Z$. Then $y z \in I$ for any $y \in I$. Now we replace $y$ with $y z$ in (2.17) and then we get

$$
\begin{aligned}
F((x \circ y) z) \pm(x \circ y) z & =F((x \circ y)) z+(x \circ y) d(z) \pm(x \circ y) z \\
& =\{F(x \circ y) \pm(x \circ y)\} z+(x \circ y) d(z) \in Z .
\end{aligned}
$$

By (2.17), we have $(x \circ y) d(z) \in Z$ that is $(x y+y x) d(z) \in Z$ for all $x, y \in I$. Now putting $y=y r$ and $x=r x, r \in R$, respectively, we obtain that $(x y r+y r x) d(z) \in Z$ and $(r x y+y r x) d(z) \in Z$. Subtracting these two results yields $[x y d(z), r] \in Z$ for all $x, y \in I$ and for all $r \in R$. This gives

$$
[[x y d(z), r], s]=0
$$

for all $x, y \in I$ and for all $r, s \in R$. We know the Jacobian identity $[[x, y], z]+[[y, z], x]+$ $[[z, x], y]=0$ for any $x, y, z \in R$. Using this identity, it follows that

$$
0=[[x y d(z), r], s]=-[[r, s], x y d(z)]-[[s, x y d(z)], r] .
$$


By using (2.19), it reduces to

$$
[[r, s], x y d(z)]=0
$$

for all $r, s \in R$ and for all $x, y \in I$. By Lemma 2.2, this implies that $[x y d(z), r]=0$, that is, $\left[I^{2} d(z), R\right]=0$. Thus $[[I, I], I d(z)]=0$ and then again by Lemma $2.2,[I, I d(z)]=0$. This yields $0=[I R, I d(z)]=I[R, I d(z)]$ which implies $I d(z) \subseteq Z$, since $[R, I d(z)] \subseteq I \cap A n n(I)=0$. Since $z$ is any nonzero element in $Z$, we conclude that $\operatorname{Id}(Z) \subseteq Z$.

\section{Acknowledgment}

The author would like to thank the referees for providing very helpful comments and suggestions.

\section{References}

[1] M. N. Daif and H. E. Bell, "Remarks on derivations on semiprime rings," International Journal of Mathematics and Mathematical Sciences, vol. 15, no. 1, pp. 205-206, 1992.

[2] M. A. Quadri, M. S. Khan, and N. Rehman, "Generalized derivations and commutativity of prime rings," Indian Journal of Pure and Applied Mathematics, vol. 34, no. 9, pp. 1393-1396, 2003.

[3] I. N. Herstein, Rings with Involution. Chicago Lectures in Mathematics, University of Chicago Press, Chicago, Ill, USA, 1976.

[4] H. E. Bell and W. S. Martindale III, "Centralizing mappings of semiprime rings," Canadian Mathematical Bulletin, vol. 30, no. 1, pp. 92-101, 1987. 


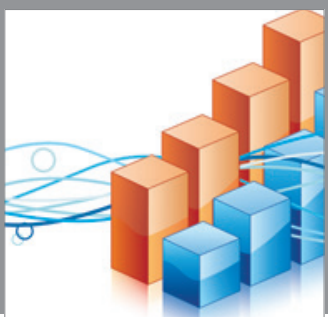

Advances in

Operations Research

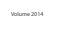

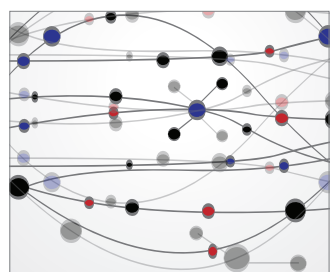

\section{The Scientific} World Journal
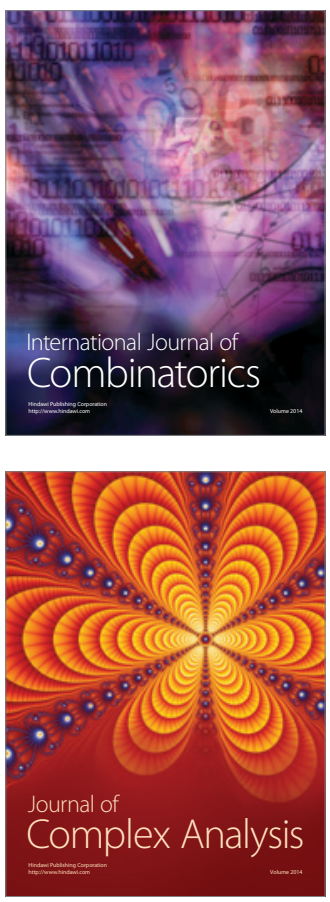

International Journal of

Mathematics and

Mathematical

Sciences
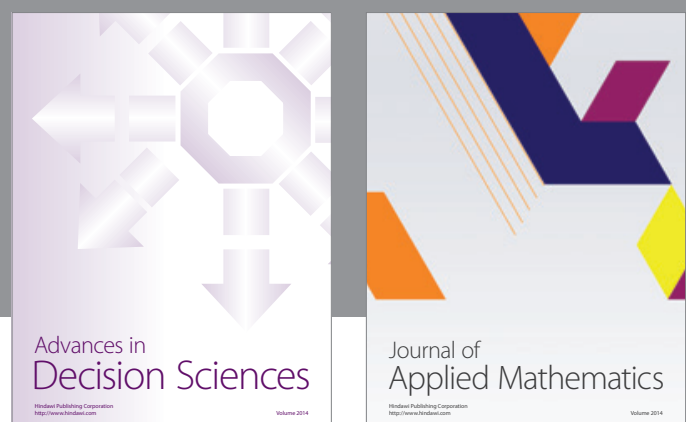

Journal of

Applied Mathematics
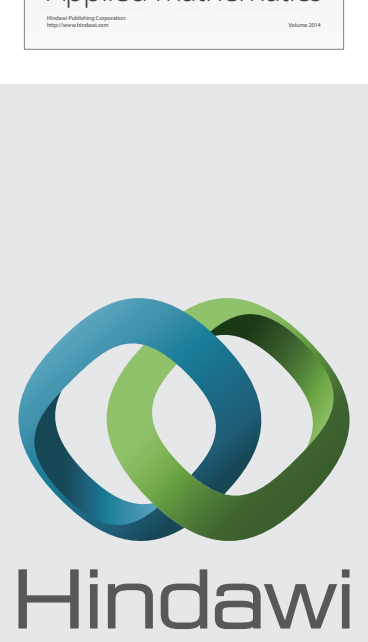

Submit your manuscripts at http://www.hindawi.com
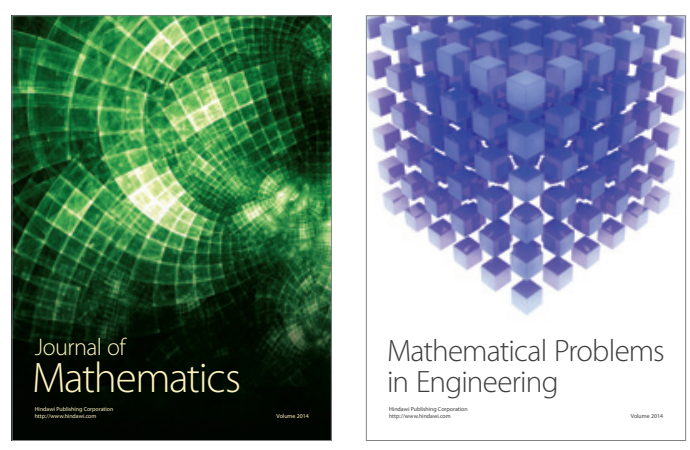

Mathematical Problems in Engineering
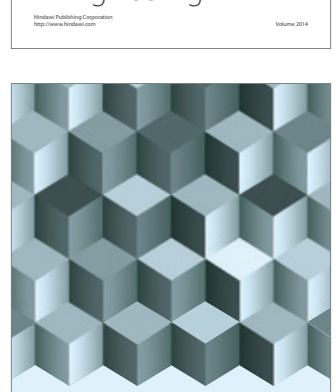

Journal of

Function Spaces
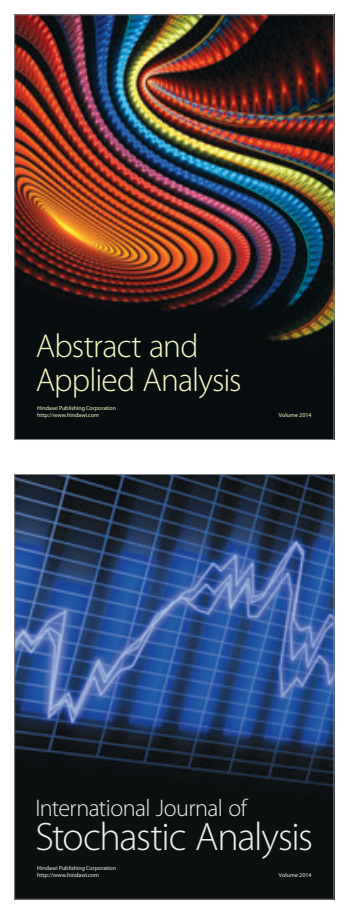

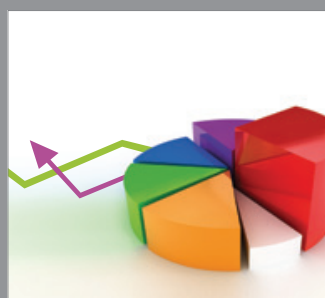

ournal of

Probability and Statistics

Promensencen
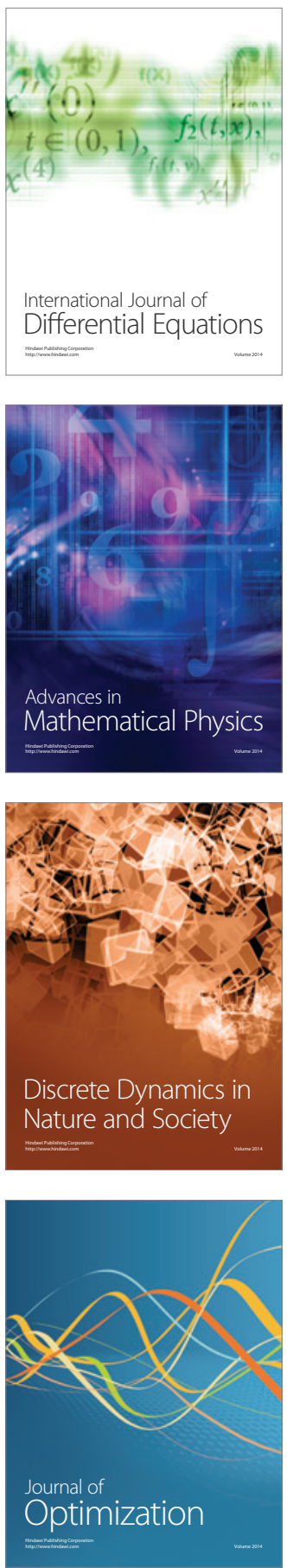